\title{
Input-output impact analysis in current or constant prices: does it matter?
}

\author{
Erik Dietzenbacher · Umed Temurshoev
}

Received: 26 March 2012 / Accepted: 27 April 2012 / Published online: 27 April 2012

(C) 2012 Dietzenbacher and Temurshoev; licensee Springer. This is an Open Access article distributed under the terms of the Creative Commons Attribution License

(http://creativecommons.org/licenses/by/2.0), which permits unrestricted use, distribution, and

reproduction in any medium, provided the original work is properly cited.

\begin{abstract}
This paper addresses the question whether the results of input-output (IO) impact analyses differ (and to what extent) when a framework in current prices or in constant prices is used. We consider the effect of an exogenous stimulus of final demand in current prices on (a) gross output in constant prices, and (b) employment. In an empirical application to Denmark, we found that all predicted effects were very similar. This holds in particular for the results at the aggregate, economy-wide level and, to a lesser extent, at the sectoral level.
\end{abstract}

Keywords input-output tables $\cdot$ impact analysis $\cdot$ current prices $\cdot$ constant prices.

JEL Classification: C67 · D57.

\section{Introduction}

Since the 1990s, physical input-output tables (PIOTs) that measure the deliveries of an industry in a single unit of mass have been compiled. In terms of appropriation of resources (e.g. water, energy, land) for final demand categories, the models based on PIOTs are an alternative to the models based on ordinary monetary inputoutput tables (MIOTs). Hubacek and Giljum (2003) were the first to compare the results for the two models. When calculating land appropriation for exports, they found substantial differences and claimed that the use of PIOTs was more appropriate. This triggered a lively discussion along two different lines. The first focused

\footnotetext{
E Dietzenbacher $(\bowtie) \cdot$ U Temurshoev

Faculty of Economics and Business, University of Groningen, PO Box 800, 9700 AV Groningen,

The Netherlands

e-mail: h.w.a.dietzenbacher@rug.nl

U Temurshoev

e-mail: u.temurshoev@rug.nl
} 
on the treatment of waste in the model based on PIOTs and was able to explain a part of the differences (see Giljum and Hubacek 2004; Giljum et al. 2004; Suh 2004; Dietzenbacher 2005; and Dietzenbacher et al. 2009).

Still, the differences remained quite substantial. Using a highly aggregated 3sector PIOT (in million tons) and MIOT (in billion DM) for Germany in 1990 (see Hubacek and Giljum 2003), the land use (in 1,000 hectares) as embodied in exports was calculated. The percentage difference for the total land appropriation amounts to $6.4 \%$ (7,281.3 thousand hectares when the MIOT based model is used versus $6,845.8$ when the PIOT based model is used). For the three underlying sectors, however, the results are quite dramatic. The percentage difference is $8.9 \%$ for the primary sector (MIOT: 6,339.3; PIOT: 5,822.4), 68.5\% for the secondary sector (MIOT: 807.1; PIOT: 478.9) and $-75.2 \%$ for the tertiary sector (MIOT: 134.9; PIOT: 544.5).

The second perspective was put forward in Weisz and Duchin (2006). They convincingly argue that the differences are caused by the fact that the two types of inputoutput (IO) tables cannot be 'translated' into each other by using a single price for all the deliveries of a given sector (or industry). Instead, deliveries from sector $i$ to sector $j$ have a different price than deliveries from sector $i$ to sector $k$ or to the final demand categories. They calculate that the implicit prices of commodity outputs (in $\mathrm{DM}$ per ton) range from 0.02 to 0.27 for the primary sector, from 0.67 to 3.80 for the secondary sector, and from 5.31 to 163.09 for the tertiary sector.

The same applies to IO tables in current and in constant prices. That is, sectors do not have a single price deflator that holds uniformly within a corresponding row of the IO table; rather, the intermediate deliveries require cell-specific deflators. This raises the question to what extent the results differ between using the model based on an IO table in current prices and the model based on an IO table in constant prices. The present paper addresses this question. In the next section, we will present the methods, one of which is novel. Section 3 discusses the results of an application using Denmark's IO tables and Section 4 concludes.

\section{Methods}

The central question is whether the results of input-output (IO) impact analyses differ (and to what extent) when a framework in current prices or in constant prices is used. To deal with this issue, we consider the following two simple cases of calculating the effect of an exogenous stimulus of final demand in current prices (a) on gross output in constant prices, and (b) on employment. Because both current and constant prices are involved, we begin with a description of the two IO frameworks.

Our starting point is an IO table in money terms (say dollars) as given in Table 1. The elements $z_{i j}$ of matrix $\mathbf{Z}$ give the domestic intermediate deliveries from sector $i$ to sector $j$, and the elements $m_{i j}$ of matrix $\mathbf{M}$ denote the imports from a foreign sector 
Table 1 Input-output table in current prices.

\begin{tabular}{llll}
\hline & Inputs & $\begin{array}{l}\text { Final demand } \\
\text { (incl gross exports) }\end{array}$ & Totals \\
\hline Domestic outputs & $\mathbf{Z}$ & $\mathbf{f}$ & $\mathbf{x}$ \\
Imports & $\mathbf{M}$ & 0 & $\mathbf{m}$ \\
Value added & $\mathbf{v}^{\prime}$ & 0 & $v$ \\
Totals & $\mathbf{x}^{\prime}$ & $f$ & \\
\hline
\end{tabular}

$i$ to sector $j .1,2$ The vector $\mathbf{f}$ with a typical element $f_{i}$ gives the domestic deliveries of sector $i$ for domestic final demand purposes (such as private consumption, private investment, government consumption and investment, and changes in stocks) and its gross exports. The row vector $\mathbf{v}^{\prime}$ with a typical element $v_{j}$ includes the value added items (such as labor payments, capital depreciation, operating surplus, and indirect taxes minus subsidies). Without loss of generality, we have assumed that there are no imported final demands (for example, imports for private consumption); neither are there any value added items in the final demand column. The vector $\mathbf{x}$ gives the domestic gross output for each sector, while $\mathbf{m}$ is the vector of total sectoral imports.

The matrix A of domestic input coefficients is obtained as $a_{i j}=z_{i j} / x_{j}$, which gives the domestic intermediate deliveries per unit of gross output. The material balance equations yield $\mathbf{x}=\mathbf{Z s}+\mathbf{f}$, where $\mathbf{s}$ indicates the summation vector consisting of ones. Using the definition of $\mathbf{A}$, these balance equations can be rewritten as $\mathbf{x}=\mathbf{A x}+\mathbf{f}$, which yields the standard Leontief model. Under the assumption that the domestic input coefficient matrix $\mathbf{A}$ remains constant, the new output vector $\mathbf{x}_{1}$ as required for an exogenously specified new final demand vector $\mathbf{f}_{1}$ is obtained as

$$
\mathbf{x}_{1}=(\mathbf{I}-\mathbf{A})^{-1} \mathbf{f}_{1}=\mathbf{L} \mathbf{f}_{1} .
$$

The matrix $\mathbf{L}=(\mathbf{I}-\mathbf{A})^{-1}$ is known as the Leontief inverse and its typical element $l_{i j}$ denotes the (additional) domestic production in dollars by sector $i$ that is required to satisfy one (extra) dollar of final demand of product $j$.

Once the gross domestic outputs are known, other factors of interest can also be computed. Suppose that information on the use of a certain factor (e.g. employment or water consumption, or land use, or $\mathrm{CO}_{2}$ emissions) is also available at the same sector classification. For example, let the element $e_{j}$ of the vector e denote the number of people employed in sector $j$. Direct employment coefficients are then obtained as $\boldsymbol{\pi}^{\prime}=\mathbf{e}^{\prime} \hat{\mathbf{x}}^{-1}$. Its element $\pi_{j}=e_{j} / x_{j}$ gives the (extra) number of employed persons per (extra) dollar of production in sector $j$. Assuming that the input matrix $\mathbf{A}$ and the direct employment coefficients are fixed, element $j$ of the row vector $\boldsymbol{\pi}^{\prime} \mathbf{L}$ then provides the (extra) number of employed people used per (extra) dollar of final de-

\footnotetext{
${ }^{1}$ Matrices are given in bold, capital letters; vectors in bold, lower case letters; and scalars in italicized, lower case letters. Vectors are columns by definition, row vectors are obtained by transposition, indicated by a prime. $\hat{\mathbf{x}}$ is a diagonal matrix with the elements of vector $\mathbf{x}$ along its main diagonal and zeros elsewhere.

${ }^{2}$ It should be stressed that it is not necessary for the full import matrix to be given. Some countries only publish the row vector that consists of the column sums of $\mathbf{M}$. This does not affect our analysis.
} 
mand for product $j$. Hence, the new employment vector $\mathbf{e}_{1}$ that is needed to satisfy the exogenous final demand vector $\mathbf{f}_{1}$ at the sector level is given by

$$
\mathbf{e}_{1}=\hat{\boldsymbol{\pi}} \mathbf{L} \mathbf{f}_{1} \text {. }
$$

The IO table in constant prices is exactly similar to Table 1, the only difference being that all flows are expressed in constant prices. To distinguish between the two types of money flows, we add a bar on the top of each matrix/vector to indicate that its elements give the constant price value. For example, the domestic intersectoral transaction matrix in constant prices is denoted by $\overline{\mathbf{Z}}$. Now assume that an analyst is interested in forecasting the values of gross outputs in constant prices and the corresponding levels of employment that are required for a new final demand $\mathbf{f}_{1}$ in current prices.

In an ideal IO world, we have that each sector $i$ produces exactly one commodity that is sold at a single price. That is, the price of commodity $i$ does not differ across buyers (i.e. any sector $j$ or final demand categories such as consumers or the government). In that case we have a single vector $\mathbf{p}$ of deflators (which are the reciprocal of the price indexes) and $\overline{\mathbf{Z}}=\hat{\mathbf{p}} \mathbf{Z}, \overline{\mathbf{x}}=\hat{\mathbf{p}} \mathbf{x}$, and $\overline{\mathbf{f}}=\hat{\mathbf{p}} \mathbf{f}$. For the matrix with input coefficients we have $\overline{\mathbf{A}}=\overline{\mathbf{Z}} \hat{\overline{\mathbf{x}}}^{-1}=\hat{\mathbf{p}} \mathbf{Z} \hat{\mathbf{x}}^{-1} \hat{\mathbf{p}}^{-1}=\hat{\mathbf{p}} \mathbf{A} \hat{\mathbf{p}}^{-1}$ and for the Leontief inverse $\overline{\mathbf{L}}=(\mathbf{I}-\overline{\mathbf{A}})^{-1}=\left(\mathbf{I}-\hat{\mathbf{p}} \mathbf{A} \hat{\mathbf{p}}^{-1}\right)^{-1}=\hat{\mathbf{p}}(\mathbf{I}-\mathbf{A})^{-1} \hat{\mathbf{p}}^{-1}=\hat{\mathbf{p}} \mathbf{L} \hat{\mathbf{p}}^{-1}$. In solving the analyst's problem from the previous paragraph, we may start from a new final demand vector $\mathbf{f}_{1}$ in current prices and first deflate it into $\hat{\mathbf{p}} \mathbf{f}_{1}$, after which the constant price IO framework is applied so as to yield $\overline{\mathbf{L}} \hat{\mathbf{p}} \mathbf{f}_{1}$. Alternatively, we may first calculate the current priced outputs required for $\mathbf{f}_{1}$ as $\mathbf{L} \mathbf{f}_{1}$, which are then deflated into $\hat{\mathbf{p}} \mathbf{L} \mathbf{f}_{1}$. Because $\overline{\mathbf{L}} \hat{\mathbf{p}}=\left(\hat{\mathbf{p}} \mathbf{L} \hat{\mathbf{p}}^{-1}\right) \hat{\mathbf{p}}=\hat{\mathbf{p}} \mathbf{L}$, the two approaches always (i.e. for any vector $\mathbf{f}_{1}$ ) yield the same answer. This implies that in an ideal world it does not matter whether the final demand vector is deflated first and then the constant price IO model is used to calculate the outputs, or whether the current price model is used first to calculate the outputs which are then deflated.

However, real world cases are quite different. Neither do sectors produce a single commodity nor do all buyers of a particular commodity pay the same price. First, sectors are aggregates of establishments that produce different commodities, which implies that sectors sell baskets of commodities and the basket sold to sector $j$ differs from the one sold to sector $k$. Due to the differences in the composition of the baskets, their prices will also be different. Second, the same commodity is often sold for prices that differ across buyers. As a consequence, deflators for the values in an IO table are typically cell-specific.

Next, we go back to the analyst's problem of forecasting the gross output values in constant prices (and the corresponding employment levels) that are required for a new final demand $\mathbf{f}_{1}$ in current prices. The two approaches sketched above yield the following.

Option A (Deflation after gross output calculations in current prices) Equation 1 gives us the predicted values of the new gross outputs in current prices. To translate these values into their constant price equivalents, one needs only to deflate the new output vector $\mathbf{x}_{1}$. In the ideal world case we used a single vector $\mathbf{p}$ of deflators. Here we use output deflators that are often available from national statistical institutes or 
that can be readily obtained from the IO table in constant prices. From the gross outputs vectors in current and constant prices, the gross output deflators are calculated as

$$
\mathbf{p}_{x}=\hat{\mathbf{x}}^{-1} \overline{\mathbf{x}}
$$

where $\overline{\mathbf{x}}$ is the gross outputs vector in constant prices. Thus, the forecasted gross outputs in constant prices are equal to

$$
\overline{\mathbf{x}}_{A}=\hat{\mathbf{p}}_{x} \mathbf{x}_{1}=\hat{\mathbf{p}}_{x} \mathbf{L} \mathbf{f}_{1},
$$

where the subscript $A$ refers to the fact that option $A$ has been used for forecasting purposes. Since we are using the IO framework in current prices, the sectoral employment forecasts $\mathbf{e}_{A}$ appear to be the same as those given in Equation 2. In a more formal fashion, let the direct coefficient of employment $\bar{\pi}_{j}$ denote the (extra) number of employed persons per (extra) gross output in constant prices in sector $j$. That is, $\overline{\boldsymbol{\pi}}^{\prime}=\mathbf{e}^{\prime} \hat{\overline{\mathbf{x}}}^{-1}$. This implies that according to option $A$ the vector of sectoral employment due to the new final demand vector $\mathbf{f}_{1}$ is derived as

$$
\mathbf{e}_{A}=\hat{\bar{\pi}} \overline{\mathbf{x}}_{A}=\hat{\mathbf{e}} \hat{\overline{\mathbf{x}}}^{-1} \hat{\mathbf{p}}_{x} \mathbf{L} \mathbf{f}_{1}=\hat{\mathbf{e}} \hat{\overline{\mathbf{x}}}^{-1}\left(\hat{\mathbf{x}}^{-1} \hat{\overline{\mathbf{x}}}\right) \mathbf{L} \mathbf{f}_{1}=\hat{\mathbf{e}} \hat{\mathbf{x}}^{-1} \mathbf{L} \mathbf{f}_{1}=\hat{\pi} \mathbf{L} \mathbf{f}_{1}=\mathbf{e}_{1}
$$

Option B (Gross output calculations in constant prices after deflation of the final demands) To use the IO setting in constant prices, one must first deflate the exogenously specified final demands in current prices, i.e. $\mathbf{f}_{1}$. Deflation can be implemented using the gross output deflators (Equation 3). However, deflators of gross outputs are in general different from those of final demands. Therefore, it is preferable to use deflators for final demands. They are usually available from statistical institutes' data, ${ }^{3}$ or - if IO tables in both current and constant prices are available - can be computed as

$$
\mathbf{p}_{f}=\hat{\mathbf{f}}^{-1} \overline{\mathbf{f}}^{.}
$$

Using the constant price domestic input coefficient matrix $\overline{\mathbf{A}}$, option $B$ 's estimate of the vector of gross outputs in constant prices is

$$
\overline{\mathbf{x}}_{B}=(\mathbf{I}-\overline{\mathbf{A}})^{-1} \hat{\mathbf{p}}_{f} \mathbf{f}_{1}=\overline{\mathbf{L}} \hat{\mathbf{p}}_{f} \mathbf{f}_{1} .
$$

Now we have two estimates of gross outputs in constant prices, namely those given in Equations 4 and 7. However, as it is evident from the corresponding equations, these estimates are in general not equal to each other. They will be exactly equal for any final demand vector $\mathbf{f}_{1}$ if and only if $\hat{\mathbf{p}}_{x} \mathbf{L}=\overline{\mathbf{L}} \hat{\mathbf{p}}_{f}$ which does not hold in real world cases. Hence, the crucial issue to consider is the significance of differences between $\overline{\mathbf{x}}_{A}$ and $\overline{\mathbf{x}}_{B}$ both at the sectoral level and at the aggregate level (i.e. after summing over the sectors).

\footnotetext{
${ }^{3}$ For example, the EU KLEMS dataset (www.euklems.net) provides price indices of gross outputs and intermediate inputs at the industry level, from which the price indices of final demands can be easily computed.
} 
For the forecasts of other factors (e.g. employment), according to option $B$, the new vector of sectoral employment due to the new final demand vector $\mathbf{f}_{1}$ is derived as

$$
\mathbf{e}_{B}=\hat{\bar{\pi}} \overline{\mathbf{x}}_{B}=\hat{\bar{\pi}} \overline{\mathbf{L}}_{f} \mathbf{f}_{1} .
$$

Using $\overline{\mathbf{x}}=\hat{\mathbf{p}}_{x} \mathbf{x}$ implies $\overline{\boldsymbol{\pi}}^{\prime}=\mathbf{e}^{\prime} \hat{\mathbf{x}}^{-1} \hat{\mathbf{p}}_{x}^{-1}=\boldsymbol{\pi}^{\prime} \hat{\mathbf{p}}_{x}^{-1}$, so that Equation 8 can be rewritten as $\mathbf{e}_{B}=\hat{\boldsymbol{\pi}} \hat{\mathbf{p}}_{x}^{-1} \overline{\mathbf{L}} \hat{\mathbf{p}}_{f} \mathbf{f}_{1}$. Note that the estimates in Equations 2 and 8 are therefore exactly the same for any vector $\mathbf{f}_{1}$ if and only if $\mathbf{L}=\hat{\mathbf{p}}_{x}^{-1} \overline{\mathbf{L}} \hat{\mathbf{p}}_{f}$. In other words, if and only if $\hat{\mathbf{p}}_{x} \mathbf{L}=\overline{\mathbf{L}} \hat{\mathbf{p}}_{f}$, which was also the necessary and sufficient condition for the equality of the gross outputs under the two options. Clearly, the two estimates in Equations 2 and 8 for employment will not match in general. Hence, it is of practical importance to find out what the size of the differences between $\mathbf{e}_{A}$ and $\mathbf{e}_{B}$ is.

Option C (Using cell-specific deflators) We consider here another option that is feasible if IO data is available both in current and constant prices. The motivation for this method stems from the fact that deflators of observed IO tables are cell-specific. It has been well documented that a single deflator does not apply uniformly within a row of the IO table (see Statistics Canada 2001). This is because a single commodity is sold for a different price to different buyers, and because sectors do not sell a single commodity but rather baskets of commodities and the composition of the baskets differs per buyer.

The proposed matching method consists of three steps. In the first step, the standard Leontief model in current prices (Equation 1) is used to derive the gross outputs vector $\mathbf{x}_{1}$ that is required for an exogenously specified final demand vector $\mathbf{f}_{1}$. In the second step, a new matrix of intermediate deliveries in current prices is calculated using the assumption that the domestic input matrix $\mathbf{A}$ is fixed. That is, $\mathbf{Z}_{1}=\mathbf{A} \hat{\mathbf{x}}_{1}$.

For the last stage, we use the cell-specific deflators as computed from the available IO data in current and constant prices. That is, the $i j$ th element of the matrix of intersectoral deflators $\mathbf{P}$ is obtained from $p_{i j}=\bar{z}_{i j} / z_{i j}$, while the final demand deflators are computed as in Equation 6. Deflating the intermediate delivery in current prices (i.e. element $z_{i j}^{1}$ of matrix $\mathbf{Z}_{1}$ ) gives $\bar{z}_{i j}^{1}=p_{i j} z_{i j}^{1}$. Thus, the sum of the deflated new intersectoral sales plus the deflated final demands gives the estimate of the gross output vector in constant prices. That is,

$$
\overline{\mathbf{x}}_{C}=\left(\mathbf{P} \otimes \mathbf{Z}_{1}\right) \mathbf{s}+\hat{\mathbf{p}}_{f} \mathbf{f}_{1},
$$

where $\otimes$ indicates the Hadamard product of element-wise multiplication.

The estimated employment vector makes use the direct employment coefficients that are expressed per gross output in constant prices, i.e.

$$
\mathbf{e}_{C}=\hat{\bar{\pi}} \overline{\mathbf{x}}_{C} \text {. }
$$

To sum up, we have now three estimates for the gross outputs in constant prices and three employment forecasts. It should be noted that option $A$ only requires an IO table in current prices and a vector of gross output deflators. Options $B$ and $C$ are more demanding in terms of data; they require the tables in current and constant prices to be available. Given the fact that in real world cases deflators do not apply uniformly within a row, option $A$ seems to be the least preferred. Options $B$ and $C$ fully use the cell-specific deflators and are thus to be preferred if data is available. 
The next section will empirically answer the question whether (and to what extent) the estimates differ from each other. We will consider the percentage differences of gross outputs and employment between the pair of methods, both at the sectoral and the total economy level. The following theorem shows that - at the sectoral level - the pairwise differences are exactly the same for gross outputs and employment (or any other factor of interest).

Theorem 1 Let $\Delta_{i}^{x}(j, k)=100 \times\left(\bar{x}_{i}^{j}-\bar{x}_{i}^{k}\right) / \bar{x}_{i}^{k}$ and $\Delta_{i}^{e}(j, k)=100 \times\left(e_{i}^{j}-e_{i}^{k}\right) / e_{i}^{k}$ be the percentage differences of, respectively, the gross output and employment estimates for sector $i$ derived by methods $j$ and $k(=A, B, C)$. Then $\Delta_{i}^{x}(j, k)=\Delta_{i}^{e}(j, k)$ holds for all $i$ and all possible combinations of two different methods $j$ and $k$.

Proof The possible pairs of methods are $A$ and $B, A$ and $C$, and $B$ and $C$. Let us start with the last combination, i.e. $j=B$ and $k=C$. First, note that comparing $\Delta_{i}^{x}(j, k)$ and $\Delta_{i}^{e}(j, k)$ is equivalent to comparing the ratios of corresponding sectoral gross outputs and sectoral employment. Let // denote Hadamard element-wise division. Then using Equations 8 and 10 we easily obtain $\mathbf{e}_{B} / / \mathbf{e}_{C}=\hat{\bar{\pi}} \overline{\mathbf{x}}_{B} / / \hat{\overline{\boldsymbol{\pi}}} \overline{\mathbf{x}}_{C}=\overline{\mathbf{x}}_{B} / / \overline{\mathbf{x}}_{C}$, which proves equivalence when methods $B$ and $C$ are compared.

Let $\mathbf{e}$ be the vector of the number of workers employed in each sector. Then Equation 3 can be written in a matrix form as $\hat{\mathbf{p}}_{x}=\hat{\mathbf{x}}^{-1} \hat{\overline{\mathbf{x}}}=\hat{\mathbf{e}} \hat{\mathbf{x}}^{-1} \hat{\mathbf{e}}^{-1} \hat{\hat{\mathbf{x}}}=\hat{\boldsymbol{\pi}} \hat{\overline{\boldsymbol{\pi}}}^{-1}$, or equivalently,

$$
\hat{\boldsymbol{\pi}}=\hat{\bar{\pi}} \hat{\mathbf{p}}_{x} .
$$

Using Equations 1, 2, 4, 5 and 11, we derive that $\mathbf{e}_{B} / / \mathbf{e}_{A}=\hat{\bar{\pi}} \overline{\mathbf{x}}_{B} / / \hat{\boldsymbol{\pi}} \mathbf{x}_{1}=\hat{\bar{\pi}} \overline{\mathbf{x}}_{B} / /$ $\hat{\bar{\pi}} \hat{\mathbf{p}}_{x} \mathbf{x}_{1}=\hat{\bar{\pi}} \overline{\mathbf{x}}_{B} / / \hat{\bar{\pi}} \overline{\mathbf{x}}_{A}=\overline{\mathbf{x}}_{B} / / \overline{\mathbf{x}}_{A}$. Going through similar steps proves that $\mathbf{e}_{C} / / \mathbf{e}_{A}=$ $\overline{\mathbf{x}}_{C} / / \overline{\mathbf{x}}_{A}$.

The result above shows that the differences between the gross outputs of any two methods provide a complete picture at the sectoral level, because the differences between the employment forecasts (or any other factor) are the same. It should be noted that by working with percentage differences, we have implicitly assumed that the employment in each sector is positive. In case the employment in sector $i$ is zero, we have that all forecasts for this sector are also zero (i.e. element $i$ of the vectors $\mathbf{e}_{A}, \mathbf{e}_{B}$, and $\mathbf{e}_{C}$ ), which is an obvious result. Finally, it should be stressed that at the economywide level the percentage difference in overall gross outputs will - in general - not be equal to that in total employment estimates. For example, comparing methods $B$ and $C$, we have for the ratio in total employment $\mathbf{s}^{\prime} \mathbf{e}_{B} / \mathbf{s}^{\prime} \mathbf{e}_{C}=\overline{\boldsymbol{\pi}}^{\prime} \overline{\mathbf{x}}_{B} / \overline{\boldsymbol{\pi}}^{\prime} \mathbf{x}_{C}$, which generally differs from $\mathbf{s}^{\prime} \mathbf{x}_{B} / \mathbf{s}^{\prime} \mathbf{x}_{C}$, the ratio in overall gross outputs.

\section{Empirical results}

We use Danish input-output (IO) tables for the period of 2000-2007 that are available from Statistics Denmark at a 130 -sector classification. ${ }^{4}$ These datasets distinguish between domestic and imported deliveries and their structure is given in Table 1. The

\footnotetext{
${ }^{4}$ The data is freely accessible from http://www.dst.dk/HomeUK/Statistics/ofs/NatAcc/IOTABLES.aspx.
} 
Table 2 Results of methods $A, B$ and $C$ for economy-wide gross output.

\begin{tabular}{|c|c|c|c|c|c|c|c|c|}
\hline & 2001 & 2002 & 2003 & 2004 & 2005 & 2006 & 2007 & Mean \\
\hline \multicolumn{9}{|c|}{ Economy-wide gross output, $n=130$} \\
\hline$(B-A) / A \%$ & 0.011 & -0.033 & -0.007 & 0.012 & 0.002 & 0.006 & -0.009 & -0.002 \\
\hline$(C-A) / A \%$ & 0.008 & -0.022 & -0.002 & 0.012 & 0.001 & 0.001 & -0.010 & -0.002 \\
\hline$(B-C) / C \%$ & 0.003 & -0.011 & -0.005 & 0.000 & 0.001 & 0.005 & 0.002 & -0.001 \\
\hline \multicolumn{9}{|c|}{ Economy-wide gross output, $n=56$} \\
\hline$(B-A) / A \%$ & 0.028 & 0.026 & 0.042 & 0.033 & 0.001 & -0.009 & -0.005 & 0.017 \\
\hline$(C-A) / A \%$ & 0.022 & 0.023 & 0.038 & 0.028 & 0.000 & -0.008 & -0.010 & 0.013 \\
\hline$(B-C) / C \%$ & 0.006 & 0.004 & 0.004 & 0.006 & 0.001 & -0.001 & 0.006 & 0.003 \\
\hline \multicolumn{9}{|c|}{ Aggregation difference: $|\Delta \%|_{n=56}-|\Delta \%|_{n=130}$} \\
\hline$(B-A) / A \%$ & 0.017 & -0.006 & 0.035 & 0.021 & -0.002 & 0.004 & -0.004 & 0.009 \\
\hline$(C-A) / A \%$ & 0.014 & 0.001 & 0.036 & 0.015 & -0.001 & 0.007 & 0.000 & 0.010 \\
\hline$(B-C) / C \%$ & 0.004 & -0.007 & -0.001 & 0.006 & -0.001 & -0.004 & 0.004 & 0.000 \\
\hline
\end{tabular}

All the percentage differences are exactly zero for year 2000 since it is the base year for the constant price IO tables. $|\Delta \%|_{n=130}$ is the absolute value of the percentage difference when the number of sectors is $n=130$. The exogenous final demand vector is the average of 2001-2007 final demand vectors in current prices, while the outcomes are gross outputs in constant prices whose overall percentage differences for methods $A, B$ and $C$ are reported.

unit of transactions is millions of Danish krones (DKK). We use the IO tables in current prices and in constant prices with the base year 2000. Further, the IO datasets are supplemented with tables showing employment figures (namely, the number of self-employed and employees, including people on a temporary leave of absence) for the 130 sectors.

We apply the tree alternatives discussed in Section 2 (i.e. options $A, B$ and $C$ ) to predict the vectors of gross outputs and employment for an exogenous final demand, which is specified as the average of the seven final demand vectors in current prices for the years 2001-2007. The computations are also done on aggregated data, where we have reduced the number of sectors from 130 to 56 . Table 2 gives the percentage differences of the results of the three methods in terms of economy-wide gross outputs (i.e. the sum of all sectoral gross outputs).

Table 2 clearly demonstrates that methods $A, B$ and $C$ provide essentially the same predictions of the economy-wide gross outputs. The reported percentage differences between the three methods are practically negligible. That is, on average, the values of overall outputs in constant prices differ from each other only by $0.001-0.002 \%$ when the number of sectors is 130 . Intuitively speaking, one might expect that the further one is in time from the base year 2000, the larger the differences are. However, this is not the case, the differences vary over time without a clear pattern.

Observe that major differences are found between $B$ and $A$ and between $C$ and $A$, whereas the differences between $B$ and $C$ are typically minor. First, note that the three types of differences are not independent of each other. That is,

$$
\frac{B-A}{A}=\frac{B-C}{A}+\frac{C-A}{A}=\frac{B-C}{C} \frac{C}{A}+\frac{C-A}{A} \approx \frac{B-C}{C}+\frac{C-A}{A}
$$


Table 3 Results of methods $A, B$ and $C$ for economy-wide employment.

\begin{tabular}{|c|c|c|c|c|c|c|c|c|}
\hline & 2001 & 2002 & 2003 & 2004 & 2005 & 2006 & 2007 & Mean \\
\hline \multicolumn{9}{|c|}{ Economy-wide employment, $n=130$} \\
\hline$(B-A) / A \%$ & 0.010 & -0.010 & 0.005 & 0.011 & -0.004 & -0.015 & -0.027 & -0.004 \\
\hline$(C-A) / A \%$ & 0.007 & -0.005 & 0.005 & 0.006 & -0.004 & -0.012 & -0.019 & -0.003 \\
\hline$(B-C) / C \%$ & 0.003 & -0.005 & 0.000 & 0.005 & 0.000 & -0.003 & -0.008 & -0.001 \\
\hline \multicolumn{9}{|c|}{ Economy-wide employment, $n=56$} \\
\hline$(B-A) / A \%$ & 0.005 & 0.000 & 0.007 & 0.022 & -0.005 & -0.012 & -0.007 & 0.001 \\
\hline$(C-A) / A \%$ & 0.002 & -0.001 & 0.004 & 0.013 & -0.004 & -0.008 & -0.005 & 0.000 \\
\hline$(B-C) / C \%$ & 0.003 & 0.002 & 0.003 & 0.009 & -0.001 & -0.005 & -0.002 & 0.001 \\
\hline \multicolumn{9}{|c|}{ Aggregation difference: $|\Delta \%|_{n=56}-|\Delta \%|_{n=130}$} \\
\hline$(B-A) / A \%$ & -0.005 & -0.010 & 0.003 & 0.011 & 0.000 & -0.003 & -0.020 & -0.003 \\
\hline$(C-A) / A \%$ & -0.005 & -0.003 & -0.001 & 0.007 & 0.000 & -0.005 & -0.014 & -0.003 \\
\hline$(B-C) / C \%$ & 0.000 & -0.003 & 0.003 & 0.004 & 0.000 & 0.002 & -0.006 & 0.000 \\
\hline
\end{tabular}

See the note to Table 2 .

because $C / A$ is approximately 1 as follows from Table 2 . If then $(B-C) / C$ is extremely close to 0 , we have that $(B-A) / A$ approximately equals $(C-A) / A$. Second, recall that methods $B$ and $C$ both use the full matrix with cell-specific deflators, whereas option $A$ only uses deflators for gross outputs and final demands.

Note that for the base year 2000, all three methods provide exactly the same outcomes since the current and constant priced IO data is exactly the same. Hence, the vectors of gross output deflators in Equation 3 and final demand deflators in Equation 6, and the matrix of intersectoral deflators $\mathbf{P}$ will consist only of ones. This implies that the vectors of predicted gross outputs in constant prices in Equations 4, 7 and 9, and employment vectors in Equations 2, 8 and 10 for methods $A, B$ and $C$, respectively, exactly coincide.

Another observation is that the aggregation from 130 to 56 sectors tends to increase the percentage differences between the methods. Still, the largest difference between gross output projections is minimal $(0.042 \%)$. So, from a practical view, aggregation from 130 to 56 sectors does not really change our conclusion. The average percentage differences ranged from 0.003 to $0.017 \%$ when 56 sectors were considered. Note that aggregation seems to affect the comparisons $B-A$ and $C-A$, but not $B-C$. This is shown by the bottom part of Table 2 with changes (going from 130 to 56 sectors) in percentage differences. Larger changes are found for the comparisons $B-A$ and $C-A$.

Table 3 provides the outcomes for total employment, which sketch a picture very similar to the results reported in Table 2 for gross output. That is, the differences between the methods are very small, they do not show a clear pattern over time, and they exhibit the same distinction (i.e. $B$ and $C$ versus $A$ ). The only difference with the finding in Table 2 is that the aggregation from 130 to 56 sectors does not tend to increase the percentage differences (on average they even decrease).

Tables 2 and 3 provided the predicted outcomes of the economy-wide output and employment. It is also interesting to see the differences at the sectoral level. The 
Table 4 Results of methods $A, B$ and $C$ for sectoral gross outputs and sectoral employment.

\begin{tabular}{|c|c|c|c|c|c|c|c|c|}
\hline & 2001 & 2002 & 2003 & 2004 & 2005 & 2006 & 2007 & Mean \\
\hline \multicolumn{9}{|c|}{$(B-A) / A \%, n=130$} \\
\hline Min & -1.278 & -2.562 & -1.586 & -0.862 & -0.588 & -1.215 & -1.708 & -1.400 \\
\hline Q1 & -0.006 & -0.031 & -0.013 & -0.005 & -0.010 & -0.018 & -0.038 & -0.017 \\
\hline Median & 0.002 & -0.006 & 0.000 & 0.003 & 0.001 & 0.000 & -0.001 & 0.000 \\
\hline Q3 & 0.011 & 0.006 & 0.013 & 0.024 & 0.022 & 0.030 & 0.035 & 0.020 \\
\hline Max & 0.472 & 0.307 & 0.348 & 0.635 & 0.520 & 1.747 & 3.297 & 1.046 \\
\hline \multicolumn{9}{|c|}{$(B-A) / A \%, n=56$} \\
\hline Min & -0.492 & -1.423 & -1.555 & -0.844 & -0.192 & -0.655 & -1.403 & -0.938 \\
\hline Q1 & -0.010 & -0.013 & -0.009 & 0.001 & -0.004 & -0.025 & -0.006 & -0.009 \\
\hline Median & 0.001 & -0.001 & 0.001 & 0.013 & 0.002 & -0.001 & 0.006 & 0.003 \\
\hline Q3 & 0.012 & 0.024 & 0.019 & 0.053 & 0.013 & 0.028 & 0.052 & 0.029 \\
\hline $\operatorname{Max}$ & 0.857 & 1.106 & 1.692 & 0.725 & 0.521 & 0.981 & 3.459 & 1.334 \\
\hline \multicolumn{9}{|c|}{$(C-A) / A \%, n=130$} \\
\hline Min & -1.321 & -2.542 & -1.565 & -0.850 & -0.584 & -1.090 & -1.395 & -1.335 \\
\hline Q1 & -0.010 & -0.021 & -0.011 & -0.008 & -0.005 & -0.014 & -0.020 & -0.013 \\
\hline Median & 0.000 & 0.000 & 0.000 & 0.000 & 0.001 & 0.000 & 0.000 & 0.000 \\
\hline Q3 & 0.007 & 0.011 & 0.014 & 0.017 & 0.020 & 0.022 & 0.028 & 0.017 \\
\hline Max & 0.463 & 0.303 & 0.346 & 0.632 & 0.490 & 1.679 & 3.280 & 1.028 \\
\hline \multicolumn{9}{|c|}{$(C-A) / A \%, n=56$} \\
\hline Min & -0.458 & -1.334 & -1.565 & -0.839 & -0.192 & -0.547 & -1.147 & -0.869 \\
\hline Q1 & -0.013 & -0.008 & -0.010 & -0.003 & -0.006 & -0.028 & -0.007 & -0.011 \\
\hline Median & 0.000 & 0.000 & -0.001 & 0.003 & 0.002 & 0.000 & 0.003 & 0.001 \\
\hline Q3 & 0.008 & 0.013 & 0.015 & 0.035 & 0.011 & 0.031 & 0.040 & 0.022 \\
\hline $\operatorname{Max}$ & 0.724 & 0.992 & 1.680 & 0.940 & 0.492 & 0.954 & 3.447 & 1.318 \\
\hline \multicolumn{9}{|c|}{$(B-C) / C \%, n=130$} \\
\hline Min & -0.055 & -0.255 & -0.214 & -0.279 & -0.071 & -0.270 & -0.409 & -0.222 \\
\hline Q1 & 0.000 & -0.010 & -0.002 & 0.000 & -0.002 & -0.005 & -0.007 & -0.004 \\
\hline Median & 0.002 & -0.003 & 0.000 & 0.003 & 0.000 & 0.000 & 0.000 & 0.000 \\
\hline Q3 & 0.006 & -0.001 & 0.001 & 0.009 & 0.002 & 0.006 & 0.005 & 0.004 \\
\hline Max & 0.070 & 0.063 & 0.069 & 0.110 & 0.123 & 0.575 & 0.868 & 0.268 \\
\hline \multicolumn{9}{|c|}{$(B-C) / C \%, n=56$} \\
\hline Min & -0.034 & -0.091 & -0.057 & -0.213 & -0.022 & -0.109 & -0.259 & -0.112 \\
\hline Q1 & 0.000 & -0.002 & 0.000 & 0.002 & -0.004 & -0.005 & -0.002 & -0.002 \\
\hline Median & 0.003 & 0.001 & 0.003 & 0.007 & -0.001 & -0.001 & 0.000 & 0.002 \\
\hline Q3 & 0.006 & 0.004 & 0.008 & 0.016 & 0.002 & 0.001 & 0.011 & 0.007 \\
\hline Max & 0.132 & 0.113 & 0.056 & 0.098 & 0.089 & 0.314 & 0.629 & 0.204 \\
\hline
\end{tabular}

distribution of these differences is described by a five-number summary of the percentage differences, i.e. the minimum, the first quartile (Q1), the median, the third quartile (Q3) and the maximum. The results are reported in Table 4, and because they 

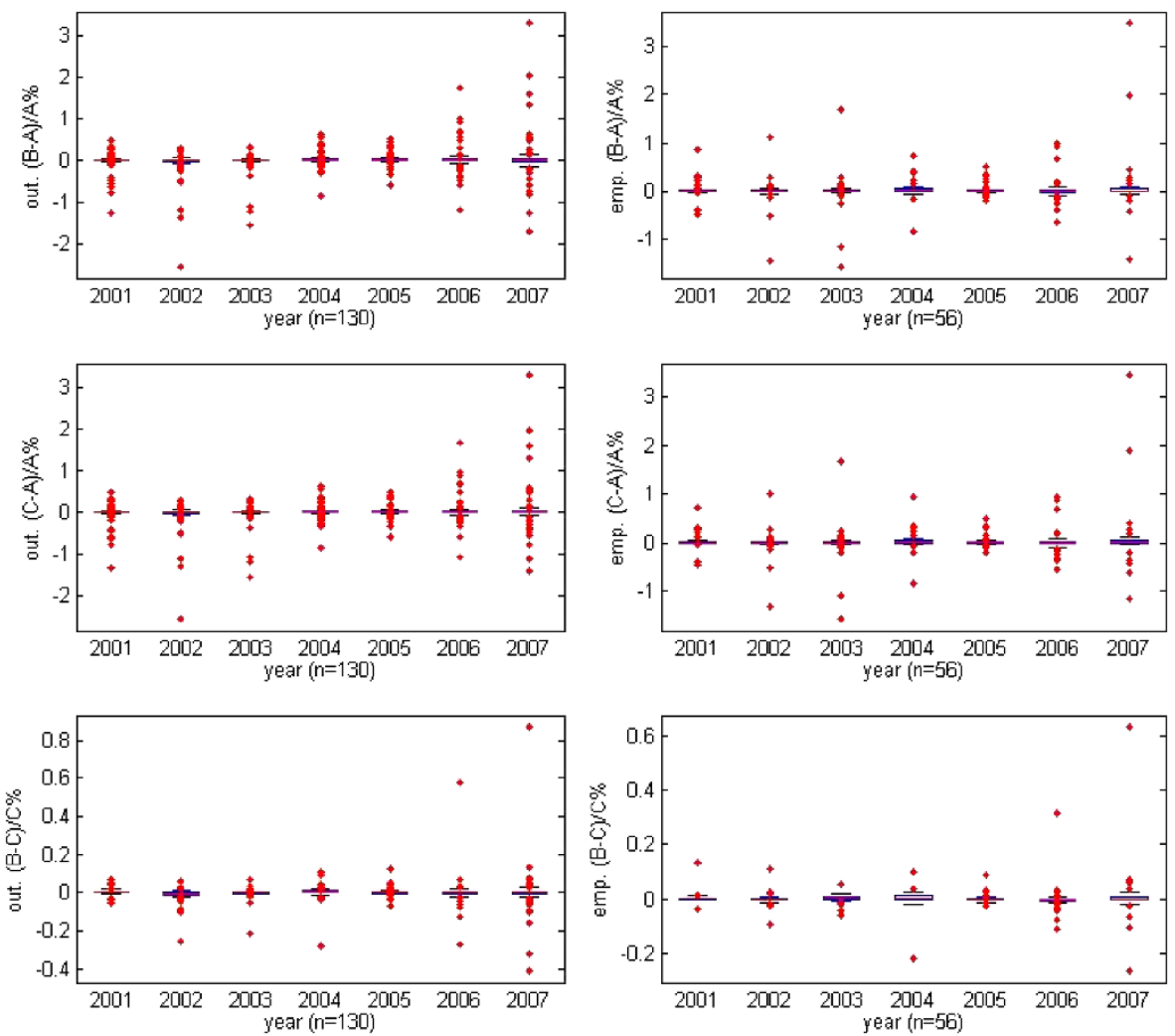

Fig. 1 Boxplots of the differences between the methods $A, B$ and $C$ at the sectoral level. In each box, the central mark is the median, the edges of the box are the 25th and 75th percentiles (i.e., first and third quartiles, Q1 and Q3), the whiskers extend to the most extreme datapoints, and the outliers are plotted individually. The extreme datapoints are determined by the so-called 1.5 interquartile range $(\mathrm{IQR}=\mathrm{Q} 3-$ $\mathrm{Q} 1)$ rule, i.e., all datapoints falling below $\mathrm{Q} 1-1.5 \times \mathrm{IQR}$ or above $\mathrm{Q} 3+1.5 \times \mathrm{IQR}$ are considered as outliers.

are at the level of individual sectors, the differences are the same for both gross outputs and employment estimates (as follows from Theorem 1). The boxplots of the same data in Figure 1 depict the full distribution of the differences.

The results at the sectoral level show that the differences for certain sectors can be much larger indeed than those at the economy-wide level. The largest percentage differences are observed in all six panels of Table 4 (or boxplots of Figure 1) for 2007. For the original data $(n=130)$, the largest difference between the results of methods $A$ and $B$ is $3.297 \%$, while it is $3.280 \%$ for $A-C$ and $0.868 \%$ for $B-C$. Similar findings hold for the aggregated data $(n=56)$. All these major differences concern the estimates of the same sector, namely, Manufacture of office machinery and computers (code 300000). This sector produced 1,885 mln DKK in 2007, which equals only $0.0617 \%$ of the Danish economy-wide gross output in current prices for that year. The corresponding percentage for gross output in constant prices is $0.0941 \%$. So, the largest differences at the sectoral level were found for a sector that was very small in terms of output. 

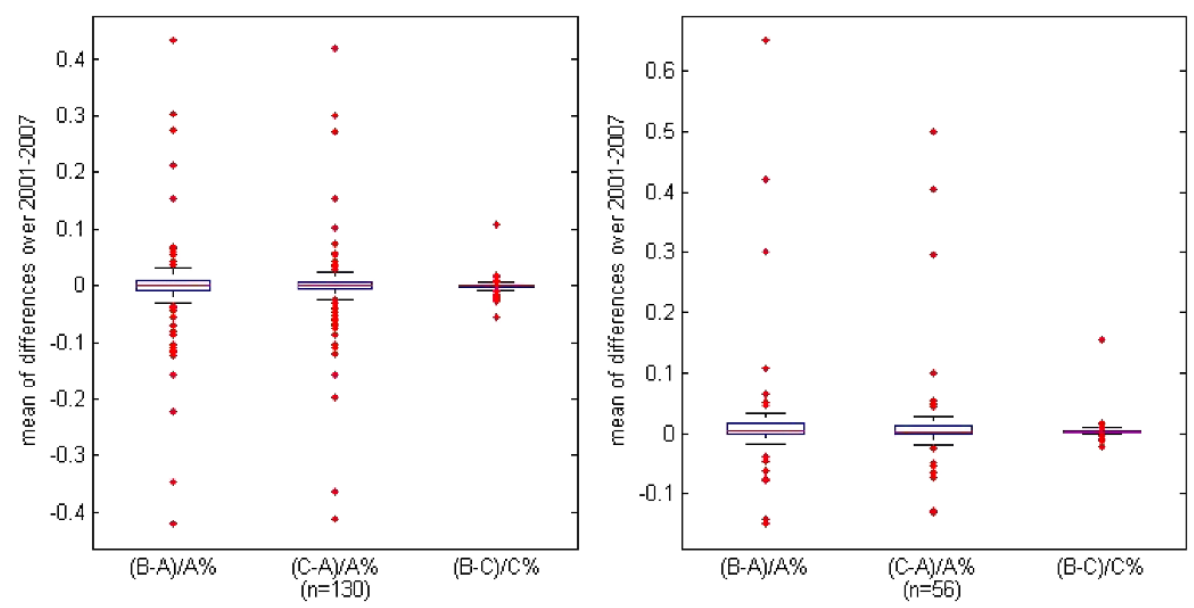

Fig. 2 Boxplots of the means over 2001-2007 for the differences between the methods at the sectoral level.

A similar finding holds for some of the other years. Again, considering the original data $(n=130)$, it turns out that Manufacture and distribution of gas (code 402000) is responsible for the largest deviation of the outcomes when comparing methods $A-B$ and $A-C$ for 2001, 2002, 2003 and 2006 (with the largest difference of $-2.562 \%$ in 2002). The shares of this sector's outputs in the overall gross outputs in current prices for these years are, respectively, $0.478,0.388,0.411$ and $0.682 \%$. The corresponding shares representing the constant price data are $0.484,0.465,0.4654$ and $0.480 \%$.

When taking the sectoral level into account, most differences between the methods are well below $1 \%$. Methods $B$ and $C$ provide estimates of both gross output in constant prices and employment that are very close to each other again. The percentage differences range from -0.409 to $0.868 \%$. For all the comparisons, we find that the outcomes when using aggregated data are very similar to those for the full 130sector data. Summarizing, there are only few sectors for which the outcomes of the different methods show a difference in the range of 1.0-3.5\% (these sectors are clear outliers in the boxplots of Figure 1). Moreover, these sectors contribute very little to the overall gross output. In all cases, their shares in gross output (both in current and constant prices) are well below $1 \%$. This explains why, for the economy-wide level figures given in Tables 2 and 3, we found such very small differences between the predictions of the three methods.

Suppose now that one is concerned about the precision of sectoral predictions. That is, suppose a 2-3\% difference is an issue even for sectors with a small contribution to the economy total output (or total employment). In that case, one might prefer methods $B$ and $C$ to method $A$. This is because the forecasts for $B$ use a domestic input matrix that is already expressed in constant prices, while $C$ uses cell-specific deflators to obtain an input matrix in constant prices. Apparently, from these two approaches, option $B$ is slightly simpler to implement. Hence, in case IO data in constant prices is available, we recommend (on the base of our findings) to use option $B$. That is, first deflate the exogenously given final demand with the final demand deflators and use the IO data in constant prices to predict gross output and/or any other factor of interest through Equations 7 and 8. 
For many purposes, however, differences of less than $5 \%$ may be neglected, in particular when they are observed for small sectors. In such cases, all three methods provide sufficiently close estimates at the sectoral level as well. In Figure 2, we provide the boxplots for the means over 2001-2007 of the percentage differences - at the sectoral level - between the methods. Both the boxplots for the aggregated and the disaggregated data show that the middle $50 \%$ of the mean percentage differences fall in an interval that has almost zero width; that most of the other datapoints (except for the outliers) fall within a range of $\pm 0.04 \%$; and that outliers range from $\pm 0.45 \%$ for the original $(n=130)$ data and from -0.15 to $0.70 \%$ for the aggregated $(n=56)$ data.

On the basis of these sectoral results and the economy-wide differences provided in Tables 2 and 3, we may conclude that, on average, the three methods considered in this paper perform very, very similarly for most practical purposes. The simplest of the three options is method $A$, for which one does not need to have IO data in constant prices (including separate deflators for final demands). That is, one can simply use the standard Leontief model in current prices to predict the sectoral gross outputs (and/or any other factor of interest) as required for an exogenously specified final demand in current prices. The obtained gross outputs then need to be deflated with the widely available gross output deflators to find the gross output estimates in constant prices.

When analyzing the differences in results obtained when using a model based on a monetary IO table with a model based on a physical IO table, Weisz and Duchin (2006) called attention to the role of prices. They argue that the common assumption is that each sector applies a single price for all of its sales. If this assumption is approximately met, the results obtained from the two models may be expected to be similar. The same also applies for the models based on IO tables in current prices and in constant prices. That is, the finding that the three methods produce outcomes that are very close to each other may to a large extent be caused by the fact that the cell-specific deflators are very much the same within each sector. If that is the case, the columns of the matrix $\mathbf{P}$ and the vectors $\mathbf{p}_{x}$ and $\mathbf{p}_{f}$ are very similar.

Table A.1 in the Appendix reports the descriptive statistics of deflators of intermediate inputs and final demand for each of 130 supplying sectors for Denmark. The year 2007 was chosen for this purpose as it is the furthest from the base year 2000. We find that for 97 sectors, the coefficient of variation (i.e. the ratio of the standard deviation and the mean) is less than $10 \%$. For the interquartile range (IQR $=\mathrm{Q} 3-\mathrm{Q} 1)$, we find that 110 sectors have IQR $<0.100$. This indicates that in 110 sectors, $50 \%$ of the sector's prices fall in a range of 0.100 (which is a small range when compared to an overall average price around one). Only 5 sectors showed an interquartile range larger than 0.200. All in all, it seems to be the case that the cell-specific deflators are fairly similar within each sector.

At the same time, it should be emphasized that the prices show quite a number of outliers. For example, the prices range from 0.105 to 8.670 in sector 4 and from 0.388 to 31.009 in sector 27 . Taking the ratio between the largest and the smallest observation for each sector, i.e. Max/Min, we find the following. For 37 sectors $\operatorname{Max} / \operatorname{Min} \geq 2.000$, in 10 of these sectors Max/Min $\geq 4.000$, and in 4 of these sectors we even find Max/Min $\geq 25.000$. In the present case, the outliers only play a marginal role because they typically are for deliveries with a small value and their effect is, thus, very small. This need not always be the case, however. 
When the cell-specific deflators have a limited variability, the situation is close to the situation with a single vector $\mathbf{p}$ of deflators. As we have seen, in such a situation there simply is no difference between applying methods $A$ and $B$ (and $C$ ). This finding is consistent with the practice of constructing tables in constant prices by applying (an adapted form of) the double deflation method (see Dietzenbacher and Hoen 1998, 1999).

\section{Concluding remarks}

In this paper we have investigated whether it matters for input-output (IO) impact analysis that the IO data is expressed in current or in constant prices. In particular, we calculated the amount of gross outputs in constant prices and employment as required for an exogenously specified vector of final demands in current prices. For this purpose we have compared three methods, all of which make use of the Leontief IO framework. These methods differed from each other in using IO data expressed: only in current prices, plus gross output deflators $(A)$; in constant prices, plus final demand deflators $(B)$; and in both current and constant prices to derive cell-specific price indices $(C)$.

We found that all three methods essentially provide very similar predictions, for the economy-wide gross output and employment in particular. For such purposes, we recommend using the simplest method $(A)$ which does not require the availability of IO data in constant prices. That is, use the standard Leontief method (based only on the IO data in current prices) to derive the estimates of gross outputs in current prices and other factors of interest (such as employment). The obtained gross outputs then need to be deflated by the widely available gross output deflators to find the corresponding outputs in constant prices.

Whenever IO data in constant prices is available, we recommend using approach $B$. That is, the vector of exogenously specified final demands in current prices first needs to be deflated by the corresponding final demand deflators (also available from various statistical dataset). Then the derived final demand stimulus vector in constant prices is used to predict the gross outputs in constant prices and any other factor using the IO framework in constant prices.

The methods $A$ and $B$ provided very similar results at the aggregate level, that is, in terms of total gross output or total employment. However, at the sectoral level the differences were somewhat larger (up to $3.5 \%$ ) for sectors with a small contribution to the total gross output. Hence, if one is worried about such deviations, then option $B$ is preferred, because the value of the intermediate deliveries between the sectors is already expressed in constant prices. Note that the aggregation of sectors (from 130 to 56) did not affect our findings.

Finally, we would like to raise three remarks, each indicating a potential direction for further research. First, all exercises in this paper used the average of the seven final demand vectors (i.e. averaged over 2001-2007) as the exogenously specified starting point. Of course, this implies that we have been working with a very specific final demand vector $\mathbf{f}_{1}$. Note that "size" does not matter for our exercise, in the sense that $k \mathbf{f}_{1}$ (for an arbitrary non-zero scalar $k$ ) would have resulted in exactly the same percentage differences between the methods. What does matter, however, and may influence the results is the sectoral mix of the final demands. A possibility for further 
investigation is not to rely on specific final demand vectors, but simply include any possibility. To this end, one should consider the full set of $n(=130,56)$ unit vectors. ${ }^{5}$ This is because any vector can be written as a linear combination of the $n$ unit vectors.

Second, Denmark was chosen because of data availability, but may of course be a very specific case. The results for other countries may be different. For example, for Denmark we found that there was only limited variability in the prices of each sector, which implies that methods $A, B$ and $C$ will generate similar results. At the same time, however, some clear outliers were observed. If these outliers occur for small deliveries, they will generate little effect. If such outliers had occurred for a few large deliveries, their influence might have been more pronounced. Clearly, this calls for an examination of a set of (preferably diverse) countries.

Third, in this paper we have restricted the aggregation to the case of $n=56 \mathrm{sec}$ tors, obtained from the original 130 sectors. The reason is that further aggregation is not recommended from an economic viewpoint (although data availability often necessitates one to aggregate further). A much more detailed analysis of the effects of aggregation might be interesting though, because there are two opposing forces at work. On the one hand, suppose that there is a uniform price for each of the original sectors (but the uniform prices differ across the sectors). Aggregation implies that the deliveries of an aggregated sector are baskets of goods produced by the original sectors and that these baskets will have a different mix of goods for different buyers (see also de Mesnard and Dietzenbacher 1995). Because the prices differ across the original sectors, the aggregated sectors are not likely to sell their products (i.e. baskets) at a uniform price. Aggregation may thus be expected to increase the discrepancies between the methods. On the other hand, if the prices of the original sectors exhibit outliers, aggregation may have a smoothening effect. In that case, the prices of some aggregated sectors may be more uniform than the original sectors and aggregation might reduce the discrepancies. An empirical analysis might provide an insight into the results of these two opposing forces at different levels of aggregation.

\section{Appendix}

Table A.1 Descriptive statistics of intermediate inputs and final demand deflators for each supplying sector (year 2007).

\begin{tabular}{llllllllllllll}
\hline Sector & 1 & 2 & 3 & 4 & 5 & 6 & 7 & 8 & 9 & 10 & 11 & 12 & 13 \\
\hline Min & 0.485 & 0.744 & 0.750 & 0.105 & 0.497 & 0.444 & 0.674 & 0.668 & 0.515 & 0.750 & 0.671 & 0.711 & 0.649 \\
Q1 & 0.920 & 0.863 & 0.870 & 0.865 & 0.808 & 1.000 & 0.805 & 0.872 & 0.821 & 0.895 & 0.855 & 0.839 & 0.834 \\
Median & 0.924 & 0.868 & 0.871 & 1.000 & 0.821 & 1.000 & 0.820 & 0.889 & 0.827 & 0.904 & 0.888 & 0.857 & 0.862 \\
Q3 & 0.933 & 0.873 & 0.900 & 1.000 & 0.846 & 1.000 & 0.914 & 0.894 & 0.830 & 0.914 & 0.911 & 0.892 & 0.902 \\
Max & 1.994 & 1.000 & 1.000 & 8.670 & 1.221 & 1.000 & 1.000 & 1.053 & 1.000 & 1.197 & 1.000 & 1.019 & 1.116 \\
Mean & 0.974 & 0.869 & 0.895 & 1.002 & 0.841 & 0.988 & 0.846 & 0.879 & 0.820 & 0.914 & 0.875 & 0.866 & 0.876 \\
Std & 0.204 & 0.024 & 0.059 & 0.791 & 0.097 & 0.073 & 0.059 & 0.051 & 0.051 & 0.065 & 0.056 & 0.048 & 0.067 \\
CV & 0.209 & 0.028 & 0.066 & 0.790 & 0.116 & 0.074 & 0.069 & 0.058 & 0.062 & 0.071 & 0.063 & 0.055 & 0.077 \\
\hline
\end{tabular}

${ }^{5}$ The $i$ th unit vector has a one on position $i$ and all other elements are zero. 
Table A.1 (Continued)

\begin{tabular}{llllllllllllll}
\hline Sector & 14 & 15 & 16 & 17 & 18 & 19 & 20 & 21 & 22 & 23 & 24 & 25 & 26 \\
\hline Min & 0.228 & 0.500 & 0.805 & 0.564 & 0.747 & 0.796 & 0.854 & 0.875 & 0.678 & 0.849 & 0.734 & 0.706 & 0.793 \\
Q1 & 0.870 & 0.809 & 1.226 & 0.879 & 0.753 & 0.866 & 0.923 & 0.927 & 0.748 & 0.942 & 0.927 & 1.098 & 1.038 \\
Median & 0.875 & 0.809 & 1.239 & 0.881 & 0.753 & 0.903 & 0.926 & 0.927 & 0.836 & 0.952 & 0.984 & 1.134 & 1.103 \\
Q3 & 0.879 & 0.811 & 1.245 & 0.884 & 0.753 & 0.922 & 0.929 & 0.927 & 0.885 & 0.968 & 1.084 & 1.146 & 1.147 \\
Max & 1.000 & 1.000 & 1.996 & 1.000 & 1.000 & 1.095 & 1.079 & 1.242 & 5.593 & 1.046 & 1.312 & 1.402 & 1.281 \\
Mean & 0.865 & 0.820 & 1.256 & 0.874 & 0.756 & 0.900 & 0.929 & 0.940 & 0.904 & 0.956 & 1.006 & 1.112 & 1.094 \\
Std & 0.068 & 0.059 & 0.196 & 0.047 & 0.022 & 0.051 & 0.027 & 0.058 & 0.583 & 0.027 & 0.116 & 0.094 & 0.096 \\
CV & 0.078 & 0.071 & 0.156 & 0.053 & 0.029 & 0.056 & 0.029 & 0.062 & 0.645 & 0.028 & 0.116 & 0.084 & 0.088 \\
\hline Sector & 27 & 28 & 29 & 30 & 31 & 32 & 33 & 34 & 35 & 36 & 37 & 38 & 39 \\
\hline
\end{tabular}

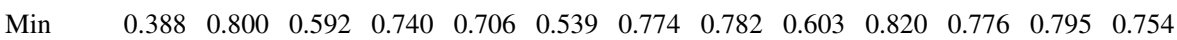

Q1 $\quad \begin{array}{llllllllllllll}0.733 & 0.818 & 0.817 & 1.000 & 0.799 & 0.777 & 0.801 & 1.199 & 0.854 & 0.868 & 0.848 & 0.887 & 0.914\end{array}$

$\begin{array}{llllllllllllll}\text { Median } & 0.788 & 0.841 & 0.819 & 1.000 & 0.816 & 0.777 & 0.808 & 1.416 & 0.874 & 0.877 & 0.867 & 0.892 & 0.940\end{array}$ Q3 $\quad \begin{array}{llllllllllllll}0.895 & 0.884 & 0.883 & 1.000 & 0.844 & 0.848 & 0.850 & 1.434 & 0.920 & 0.883 & 0.878 & 0.901 & 0.950\end{array}$

$\begin{array}{llllllllllllll}\operatorname{Max} & 31.009 & 1.116 & 1.209 & 1.000 & 1.180 & 1.098 & 1.000 & 1.445 & 1.129 & 1.000 & 1.354 & 1.239 & 1.304\end{array}$ $\begin{array}{lllllllllllllll}\text { Mean } & 1.298 & 0.860 & 0.864 & 0.961 & 0.824 & 0.814 & 0.830 & 1.296 & 0.890 & 0.880 & 0.872 & 0.898 & 0.930\end{array}$ $\begin{array}{llllllllllllll}\text { Std } & 3.098 & 0.057 & 0.088 & 0.093 & 0.069 & 0.094 & 0.046 & 0.192 & 0.068 & 0.024 & 0.060 & 0.045 & 0.074\end{array}$ $\begin{array}{lllllllllllllll}\mathrm{CV} & 2.387 & 0.067 & 0.102 & 0.097 & 0.083 & 0.115 & 0.055 & 0.148 & 0.076 & 0.027 & 0.069 & 0.050 & 0.079\end{array}$

\begin{tabular}{llllllllllllll}
\hline Sector & 40 & 41 & 42 & 43 & 44 & 45 & 46 & 47 & 48 & 49 & 50 & 51 & 52 \\
\hline Min & 0.674 & 0.744 & 0.381 & 0.423 & 0.631 & 0.700 & 0.463 & 0.518 & 0.418 & 0.676 & 0.774 & 0.728 & 0.422 \\
Q1 & 0.806 & 0.866 & 0.798 & 0.752 & 0.793 & 0.801 & 0.806 & 0.820 & 0.804 & 0.909 & 0.935 & 0.886 & 0.882 \\
Median & 0.806 & 0.901 & 0.881 & 0.804 & 0.863 & 0.915 & 0.851 & 0.869 & 0.806 & 0.927 & 0.938 & 0.924 & 0.940 \\
Q3 & 0.806 & 0.924 & 0.896 & 0.840 & 0.873 & 0.927 & 0.854 & 0.892 & 0.827 & 0.932 & 0.939 & 0.934 & 0.943 \\
Max & 1.000 & 1.000 & 1.000 & 1.000 & 1.000 & 1.000 & 1.000 & 1.107 & 1.000 & 1.000 & 1.000 & 1.080 & 1.134 \\
Mean & 0.809 & 0.892 & 0.817 & 0.792 & 0.832 & 0.872 & 0.831 & 0.861 & 0.816 & 0.913 & 0.930 & 0.909 & 0.906 \\
Std & 0.058 & 0.041 & 0.132 & 0.070 & 0.078 & 0.070 & 0.052 & 0.060 & 0.063 & 0.039 & 0.025 & 0.059 & 0.088 \\
CV & 0.072 & 0.046 & 0.161 & 0.088 & 0.093 & 0.080 & 0.063 & 0.070 & 0.077 & 0.043 & 0.027 & 0.064 & 0.097 \\
\hline Sector & 53 & 54 & 55 & 56 & 57 & 58 & 59 & 60 & 61 & 62 & 63 & 64 & 65 \\
\hline Min & 0.840 & 0.735 & 0.754 & 0.711 & 0.406 & 0.670 & 0.792 & 0.704 & 0.755 & 0.847 & 0.464 & 0.317 & 0.067 \\
Q1 & 0.851 & 0.912 & 0.952 & 0.881 & 0.914 & 0.918 & 0.927 & 0.848 & 0.890 & 0.848 & 0.519 & 0.635 & 0.894 \\
Median & 0.852 & 0.931 & 0.953 & 0.916 & 0.931 & 0.920 & 0.937 & 0.915 & 0.928 & 0.848 & 0.559 & 1.000 & 0.894 \\
Q3 & 0.885 & 0.935 & 0.971 & 0.938 & 0.937 & 0.920 & 0.939 & 0.927 & 0.932 & 0.848 & 0.820 & 1.114 & 1.000 \\
Max & 1.529 & 1.063 & 1.745 & 1.354 & 1.000 & 1.000 & 1.402 & 4.959 & 1.000 & 1.000 & 1.578 & 1.663 & 4.810 \\
Mean & 0.901 & 0.921 & 0.967 & 0.916 & 0.914 & 0.907 & 0.932 & 0.955 & 0.910 & 0.849 & 0.693 & 0.901 & 0.996 \\
Std & 0.124 & 0.042 & 0.094 & 0.070 & 0.060 & 0.045 & 0.049 & 0.424 & 0.043 & 0.013 & 0.227 & 0.261 & 0.491 \\
CV & 0.138 & 0.046 & 0.098 & 0.076 & 0.065 & 0.050 & 0.053 & 0.444 & 0.047 & 0.016 & 0.328 & 0.290 & 0.493 \\
\hline
\end{tabular}


Table A.1 (Continued)

\begin{tabular}{llllllllllllll}
\hline Sector & 66 & 67 & 68 & 69 & 70 & 71 & 72 & 73 & 74 & 75 & 76 & 77 & 78 \\
\hline Min & 0.720 & 0.833 & 0.779 & 0.775 & 0.790 & 0.743 & 0.818 & 0.556 & 0.449 & 0.728 & 0.777 & 0.692 & 0.527 \\
Q1 & 0.726 & 1.000 & 0.779 & 0.787 & 0.813 & 0.819 & 0.836 & 0.808 & 0.749 & 0.823 & 0.941 & 0.931 & 0.960 \\
Median & 0.726 & 1.000 & 0.779 & 0.797 & 0.813 & 0.850 & 0.836 & 0.889 & 0.845 & 0.857 & 1.003 & 0.965 & 0.960 \\
Q3 & 0.726 & 1.000 & 0.779 & 0.835 & 0.813 & 0.888 & 0.836 & 1.004 & 0.897 & 0.899 & 1.035 & 1.007 & 0.960 \\
Max & 1.000 & 1.000 & 1.000 & 1.000 & 1.000 & 1.902 & 1.000 & 2.611 & 1.085 & 1.818 & 2.266 & 17.723 & 1.787 \\
Mean & 0.735 & 0.999 & 0.785 & 0.812 & 0.817 & 0.873 & 0.838 & 0.936 & 0.829 & 0.866 & 0.997 & 1.103 & 0.945 \\
Std & 0.047 & 0.015 & 0.033 & 0.035 & 0.028 & 0.122 & 0.020 & 0.247 & 0.110 & 0.111 & 0.140 & 1.466 & 0.097 \\
CV & 0.064 & 0.015 & 0.043 & 0.043 & 0.035 & 0.140 & 0.024 & 0.263 & 0.133 & 0.128 & 0.140 & 1.329 & 0.103 \\
\hline Sector & 79 & 80 & 81 & 82 & 83 & 84 & 85 & 86 & 87 & 88 & 89 & 90 & 91 \\
\hline
\end{tabular}

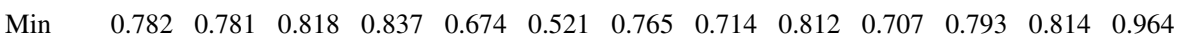
Q1 $\quad \begin{array}{lllllllllllll}0.891 & 0.825 & 0.839 & 0.842 & 0.679 & 0.738 & 0.781 & 0.727 & 0.895 & 0.852 & 0.795 & 1.002 & 1.238\end{array}$

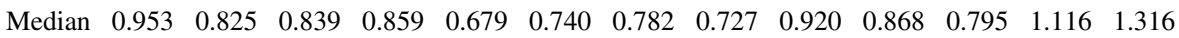
Q3 $\quad \begin{array}{lllllllllllllll}1.016 & 0.825 & 0.842 & 0.906 & 0.679 & 0.744 & 0.785 & 0.727 & 0.941 & 0.890 & 1.000 & 1.162 & 1.373\end{array}$

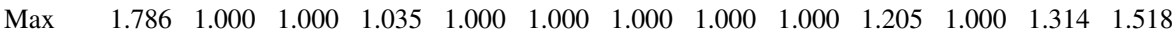

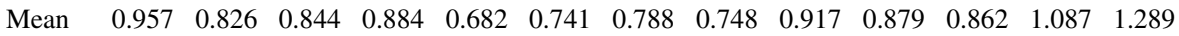
$\begin{array}{llllllllllllll}\text { Std } & 0.109 & 0.020 & 0.021 & 0.055 & 0.028 & 0.036 & 0.027 & 0.072 & 0.035 & 0.053 & 0.097 & 0.109 & 0.127\end{array}$ $\begin{array}{llllllllllllll}\mathrm{CV} & 0.114 & 0.024 & 0.025 & 0.062 & 0.041 & 0.048 & 0.035 & 0.096 & 0.038 & 0.060 & 0.112 & 0.100 & 0.098\end{array}$

\begin{tabular}{llllllllllllll}
\hline Sector & 92 & 93 & 94 & 95 & 96 & 97 & 98 & 99 & 100 & 101 & 102 & 103 & 104 \\
\hline
\end{tabular}

$\begin{array}{llllllllllllll}\text { Min } & 0.913 & 1.000 & 1.000 & 0.911 & 0.381 & 0.815 & 0.862 & 0.874 & 0.821 & 0.994 & 0.796 & 0.800 & 0.821\end{array}$ Q1 $\quad \begin{array}{llllllllllllll}1.126 & 1.000 & 1.074 & 0.914 & 0.382 & 1.000 & 0.863 & 0.962 & 0.948 & 0.999 & 0.798 & 0.856 & 0.830\end{array}$ $\begin{array}{llllllllllllll}\text { Median } & 1.259 & 1.000 & 1.076 & 0.915 & 0.382 & 1.000 & 0.863 & 1.037 & 0.985 & 1.000 & 1.000 & 0.857 & 0.830\end{array}$ Q3 $\quad \begin{array}{llllllllllllll}1.330 & 1.000 & 1.078 & 0.918 & 0.382 & 1.000 & 0.863 & 1.101 & 0.999 & 1.001 & 1.000 & 0.859 & 0.830\end{array}$ $\begin{array}{llllllllllllll}\operatorname{Max} & 1.506 & 1.073 & 1.085 & 1.000 & 1.000 & 1.000 & 1.000 & 1.739 & 1.011 & 1.005 & 2.303 & 1.000 & 1.989\end{array}$ $\begin{array}{llllllllllllll}\text { Mean } & 1.229 & 1.001 & 1.074 & 0.919 & 0.387 & 0.999 & 0.869 & 1.037 & 0.968 & 1.000 & 1.128 & 0.868 & 0.841\end{array}$ $\begin{array}{lllllllllllllll}\text { Std } & 0.148 & 0.009 & 0.012 & 0.017 & 0.054 & 0.016 & 0.027 & 0.119 & 0.042 & 0.002 & 0.484 & 0.040 & 0.103\end{array}$

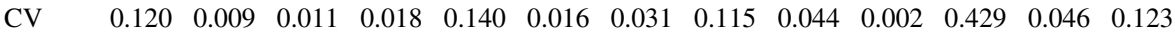

\begin{tabular}{llllllllllllll}
\hline Sector & 105 & 106 & 107 & 108 & 109 & 110 & 111 & 112 & 113 & 114 & 115 & 116 & 117 \\
\hline Min & 0.762 & 0.749 & 1.000 & 0.777 & 0.780 & 0.826 & 0.829 & 0.750 & 0.762 & 0.826 & 0.814 & 0.818 & 0.500 \\
Q1 & 0.762 & 0.790 & 1.169 & 0.791 & 0.793 & 0.846 & 0.838 & 0.874 & 0.782 & 1.000 & 1.000 & 0.859 & 0.662 \\
Median & 0.762 & 0.795 & 1.171 & 0.792 & 0.796 & 0.846 & 0.838 & 0.875 & 0.811 & 1.000 & 1.000 & 0.859 & 0.662 \\
Q3 & 0.762 & 0.821 & 1.172 & 0.793 & 0.798 & 0.847 & 0.839 & 0.875 & 0.841 & 1.000 & 1.000 & 0.859 & 0.663 \\
Max & 1.000 & 1.000 & 2.048 & 1.000 & 1.000 & 1.000 & 1.000 & 1.000 & 1.000 & 1.000 & 1.000 & 1.000 & 1.992 \\
Mean & 0.766 & 0.810 & 1.172 & 0.796 & 0.799 & 0.853 & 0.855 & 0.877 & 0.815 & 0.971 & 0.969 & 0.870 & 0.772 \\
Std & 0.029 & 0.034 & 0.083 & 0.023 & 0.025 & 0.030 & 0.040 & 0.025 & 0.041 & 0.064 & 0.068 & 0.040 & 0.327 \\
CV & 0.038 & 0.041 & 0.070 & 0.029 & 0.031 & 0.035 & 0.047 & 0.028 & 0.050 & 0.065 & 0.071 & 0.046 & 0.423 \\
\hline
\end{tabular}


Table A.1 (Continued)

\begin{tabular}{llllllllllllll}
\hline Sector & 118 & 119 & 120 & 121 & 122 & 123 & 124 & 125 & 126 & 127 & 128 & 129 & 130 \\
\hline Min & 0.839 & 0.823 & 0.726 & 0.818 & 0.826 & 0.685 & 0.781 & 0.778 & 0.821 & 0.654 & 0.844 & 0.769 & 0.775 \\
Q1 & 0.854 & 1.000 & 0.903 & 1.000 & 1.000 & 0.693 & 0.782 & 0.782 & 0.823 & 0.787 & 1.000 & 0.878 & 1.000 \\
Median & 0.854 & 1.000 & 0.905 & 1.000 & 1.000 & 0.698 & 0.782 & 0.782 & 0.823 & 0.787 & 1.000 & 0.882 & 1.000 \\
Q3 & 0.855 & 1.000 & 0.905 & 1.000 & 1.000 & 0.707 & 0.782 & 0.782 & 0.823 & 0.976 & 1.000 & 0.883 & 1.000 \\
Max & 1.000 & 1.000 & 1.000 & 1.000 & 1.000 & 1.000 & 1.000 & 1.000 & 1.000 & 1.902 & 1.000 & 1.000 & 1.000 \\
Mean & 0.859 & 0.974 & 0.888 & 0.969 & 0.971 & 0.705 & 0.797 & 0.802 & 0.826 & 0.939 & 0.981 & 0.876 & 0.998 \\
Std & 0.028 & 0.056 & 0.037 & 0.067 & 0.062 & 0.038 & 0.055 & 0.063 & 0.022 & 0.284 & 0.041 & 0.024 & 0.020 \\
CV & 0.033 & 0.058 & 0.042 & 0.069 & 0.064 & 0.054 & 0.070 & 0.079 & 0.027 & 0.303 & 0.041 & 0.028 & 0.020 \\
\hline
\end{tabular}

Std stands for standard deviation, CV for coefficient of variation.

\section{Competing interests}

The authors declare that they have no competing interests.

Acknowledgements We gratefully acknowledge financial support provided by EU FP7 WIOD project. This project is funded by the European Commission, Research Directorate General as part of the 7th Framework Programme, Theme 8: Socio-Economic Sciences and Humanities. Grant Agreement no: 225 281, www.wiod.org.

\section{References}

de Mesnard L, Dietzenbacher E (1995) On the interpretation of fixed input coefficients under aggregation. J Reg Sci 35:231-241

Dietzenbacher E (2005) Waste treatment in physical input-output analysis. Ecol Econ 55:11-23

Dietzenbacher E, Giljum S, Hubacek K, Suh S (2009) Physical input-output analysis and disposals to nature. In: Suh S (ed) Handbook of input-output economics in industrial ecology. Springer, Heidelberg, pp 123-137

Dietzenbacher E, Hoen AR (1998) Deflation of input-output tables from the user's point of view: a heuristic approach. Rev Income Wealth 44:111-122

Dietzenbacher E, Hoen AR (1999) Double deflation and aggregation. Environ Plan A 31:1695-1704

Giljum S, Hubacek K (2004) Alternative approaches of physical input-output analysis to estimate primary material inputs of production and consumption activities. Econ Syst Res 16:301-310

Giljum S, Hubacek K, Sun L (2004) Beyond the simple material balance: a reply to Sangwon Suh's note on physical input-output analysis. Ecol Econ 48:19-22

Hubacek K, Giljum S (2003) Applying physical input-output analysis to estimate land appropriation (ecological footprints) of international trade activities. Ecol Econ 44:137-151

Statistics Canada (2001) A guide to deflating the input-output accounts: sources and methods. Catalogue No $15 \mathrm{~F} 0077 \mathrm{GIE}$

Suh S (2004) A note on the calculus for physical input-output analysis and its applications to land appropriation of international trade activities. Ecol Econ 48:9-17

Weisz H, Duchin F (2006) Physical and monetary input-output analysis: what makes the difference? Ecol Econ 57:534-541 